\title{
Natural Vibrations of the Reinforced Tapered Shell with Taking Into Account the Viscoelastic Properties of the Material
}

\author{
Matlab Ishmamatov $^{1}$, Nurillo Kulmuratov ${ }^{1 *}$, Nasriddin Axmedov ${ }^{1}$, Shaxob Xalilov ${ }^{1}$, \\ Sherzod Ablakulov ${ }^{2}$ \\ ${ }^{1}$ Navoi State Mining Institute, Navoi, Uzbekistan \\ ${ }^{2}$ Tashkent Institute of Chemical Technology, Tashkent, Uzbekistan
}

\begin{abstract}
In this paper, the integro-differential equations of natural oscillations of a viscoelastic ribbed truncated conical shell are obtained based on the Lagrange variational equation. The general research methodology is based on the variational principles of mechanics and variational methods. Geometrically nonlinear mathematical models of the deformation of ribbed conical shells are obtained, considering such factors as the discrete introduction of edges. Based on the finite element method, a method for solving and an algorithm for the equations of natural oscillations of a viscoelastic ribbed truncated conical shell with articulated and freely supported edges is developed. The problem is reduced to solving homogeneous algebraic equations with complex coefficients of large order. For a solution to exist, the main determinant of a system of algebraic equations must be zero. From this condition, we obtain a frequency equation with complex output parameters. The study of natural vibrations of viscoelastic panels of truncated conical shells is carried out, and some characteristic features are revealed. The complex roots of the frequency equation are determined by the Muller method. At each iteration of the Muller method, the Gauss method is used with the main element selection. As the number of edges increases, the real and imaginary parts of the eigenfrequencies increase, respectively.
\end{abstract}

\section{Introduction}

Conical shell structures are widely used in rocketry, aircraft construction, shipbuilding, and construction. To give greater rigidity, the thin-walled part of the shell is reinforced with ribs, while a slight increase in the weight of the structure significantly increases its strength, even if the ribs are of low height.

Of great practical interest is the study and elimination of resonant phenomena in shells. A considerable number of theoretical and experimental works have been devoted to the study of the natural oscillations of circular cones. However, there are still no reliable

*Corresponding author: nurillo.kulmuratov.64@mail.ru 
solutions that allow us to determine the parameters of resonances in a wide range of changes in physical and geometric parameters. There are also works in which the dependences for determining the resonant frequencies [1] and the vibration forms of truncated conical panels [2,3] are obtained by theoretical and experimental methods. Another method is mainly used to study shells, which allows us to move from the stability equations of conical shells to the corresponding equations for cylindrical shells with a circular cross-section. In many works, the non-momentary and semi-momentary theory of shells is used [4,5]. Approximate methods are also used to solve problems of natural oscillations [6,7]. A particular difficulty is caused by the problems of vibrations of reinforced conical shells in a geometrically nonlinear formulation, taking into account the material's rheological properties, for which there are practically no solutions. The literature analysis shows that the existing optimal shell designs for the given geometric and rheological parameters cannot be implemented in practice; the level of research remains only theoretical. In this regard, despite the long history of solving the problem, the determination of the resonant frequency of natural oscillations, taking into account the structural properties of ribbed shells, remains relevant. The results obtained in this paper can be directly used in practice in dynamic calculations and the design of structures whose elements are ribbed shells of rotation. The aim of this work is to develop a method, algorithm, and program for finding resonant frequencies and vibration forms for circular ribbed viscoelastic conical shells under various boundary conditions.

\section{Methods}

\subsection{Problem statement and basic relations}

Consider a closed circular conic shell with a taper angle $\theta$, with thick $h$ (Fig. 1) with edges 1 and $n$ (in the longitudinal and annular directions, respectively). To obtain the equations of natural oscillations, we use the principle of possible Lagrange displacements, which takes into account the boundary conditions

$$
\delta(\mathrm{K}+\Pi-\mathrm{A})=0
$$

where $\mathrm{K}$ is the kinetic energy of the shell and the edge, $\mathrm{P}$ is the potential energy of the shell and the edge, and A is the work of external forces. The median surface of the shell is taken as the coordinate surface. Axes X, Y The orthogonal coordinate system, directed along the lines of the main curvature, is shown in Figure 1, and the axis is directed orthogonally to the median surface in the direction of concavity. For a conic shell, the Lame and curvature parameters given in [8] take the following form $\mathrm{A}=1, \mathrm{~B}=\mathrm{x} \sin \theta$,

$$
\mathrm{k}_{\mathrm{x}}=0, \mathrm{k}_{\mathrm{y}}=\frac{\operatorname{ctg} \theta}{\mathrm{x}} \text {. }
$$




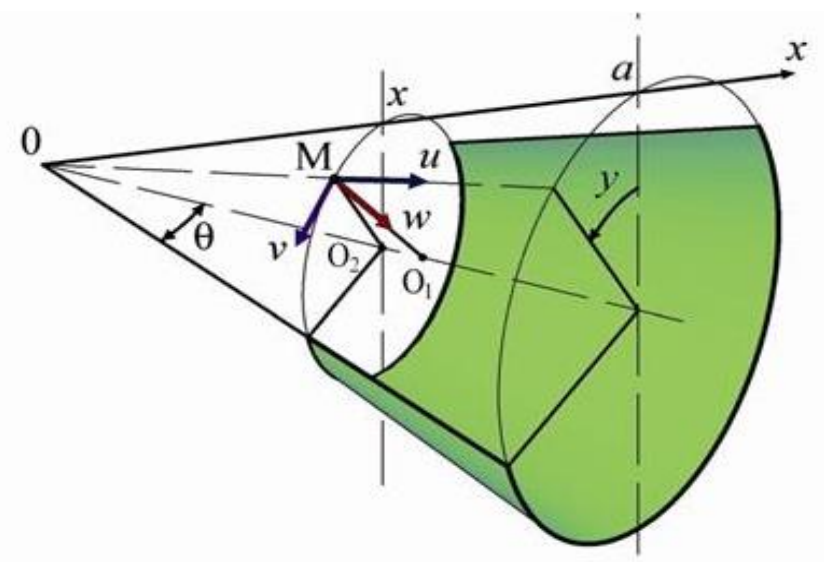

Fig.1. The truncated conical shell with reinforced ribs

Deformations in the coordinate surface of the shell are expressed in terms of displacements $\mathrm{u}, \mathrm{v}, \mathrm{w}$ along the axes $\mathrm{x}, \mathrm{y}, \mathrm{z}$ accordingly, as follows

$$
\begin{gathered}
\varepsilon_{\mathrm{xx}}=\frac{\partial \mathrm{u}}{\partial \mathrm{x}}+\frac{1}{2} \cdot\left(\frac{\partial \mathrm{w}}{\partial \mathrm{x}}\right)^{2} \\
\varepsilon_{\mathrm{yy}}=\frac{1}{\mathrm{x} \sin \theta} \cdot \frac{\partial \mathrm{v}}{\partial \mathrm{y}}+\frac{\mathrm{u}}{\mathrm{x}}-\frac{\operatorname{ctg} \theta}{\mathrm{x}} \mathrm{w}+\frac{1}{2} \cdot\left(\frac{1}{\mathrm{x} \sin \theta} \cdot \frac{\partial \mathrm{w}}{\partial \mathrm{y}}+\frac{\operatorname{ctg} \theta}{\mathrm{x}} \mathrm{v}\right)^{2} \\
\gamma_{\mathrm{xy}}=\frac{\partial \mathrm{v}}{\partial \mathrm{x}}+\frac{1}{\mathrm{x} \sin \theta} \cdot \frac{\partial \mathrm{u}}{\partial \mathrm{y}}-\frac{\mathrm{v}}{\mathrm{x}}+\frac{\partial \mathrm{w}}{\partial \mathrm{x}} \cdot\left(\frac{1}{\mathrm{x} \sin \theta} \cdot \frac{\partial \mathrm{v}}{\partial \mathrm{y}}+\frac{\operatorname{ctg} \theta}{\mathrm{x}} \mathrm{v}\right)
\end{gathered}
$$

Deformations in a layer spaced at a distance of $\mathrm{z}$ from the coordinate surface, taking into account the transverse shifts, have the form

$$
\begin{gathered}
\left(u^{\mathrm{z}}=\mathrm{u}+\mathrm{z} \cdot \psi_{\mathrm{x}}, \mathrm{v}^{\mathrm{z}}=\mathrm{v}+\mathrm{z} \psi_{\mathrm{y}}, \mathrm{w}^{\mathrm{z}}=\mathrm{w}\right) \\
\varepsilon_{\mathrm{x}}^{\mathrm{z}}=\varepsilon_{\mathrm{xx}}+\mathrm{z} \cdot \chi_{1} \quad ; \varepsilon_{\mathrm{y}}^{\mathrm{z}}=\varepsilon_{\mathrm{yy}}+\mathrm{z} \cdot \chi_{2} \quad ; \gamma_{\mathrm{xy}}^{\mathrm{z}}=\gamma_{\mathrm{xy}}+2 \cdot \mathrm{z} \cdot \chi_{12}
\end{gathered}
$$

and in addition

$$
\gamma_{\mathrm{xz}}=\mathrm{kg}(\mathrm{z}) \cdot\left(\Psi_{\mathrm{x}}+\frac{\partial \mathrm{w}}{\partial \mathrm{x}}\right) ; \gamma_{\mathrm{yz}}=\mathrm{kg}(\mathrm{z}) \cdot\left(\psi_{\mathrm{y}}+\frac{1}{\mathrm{x} \sin \theta} \cdot \frac{\partial \mathrm{w}}{\partial \mathrm{y}}+\operatorname{ctg} \theta \frac{\mathrm{v}}{\mathrm{x}}\right)
$$

Here $\psi_{\mathrm{x}}, \psi_{\mathrm{y}}$ are the angles of rotation of the normal segment at the coordinate surface in sections XOZ and YOZ accordingly; $\mathrm{g}(\mathrm{z})$ is function describing the distribution $\tau_{\mathrm{xz}}$ and $\tau_{\mathrm{yz}}$ tangential stresses; $\mathrm{k}=$ const. $[10]$

Functions that characterize changes in curvature $\chi_{1}, \chi_{2}$ and torsion $\chi_{12}$ have the form

$$
\begin{aligned}
& \chi_{1}=\frac{\partial \Psi_{x}}{\partial x} ; \chi_{2}=\frac{1}{x \sin \theta} \cdot \frac{\partial \psi_{y}}{\partial y}+\frac{\Psi_{x}}{x} \\
& 2 \chi_{12}=\frac{\partial \Psi_{y}}{\partial x}+\frac{1}{x \sin \theta} \cdot \frac{\partial \Psi_{x}}{\partial y}-\frac{\psi_{y}}{x}
\end{aligned}
$$

The physical relations for an isotropic viscoelastic body take the form [11] 


$$
\begin{gathered}
\sigma_{\mathrm{x}}=\frac{\widetilde{\mathrm{E}}}{1-v^{2}}\left(\varepsilon_{\mathrm{x}}^{\mathrm{z}}+v \varepsilon_{\mathrm{y}}^{\mathrm{z}}\right) ; \sigma_{\mathrm{y}}=\frac{\widetilde{\mathrm{E}}}{1-v^{2}} \cdot\left(\varepsilon_{\mathrm{y}}^{\mathrm{z}}+v \varepsilon_{\mathrm{x}}^{\mathrm{z}}\right) ; \\
\tau_{\mathrm{xy}}=\frac{\widetilde{\mathrm{E}}}{2(1+v)} \gamma_{\mathrm{xy}}^{\mathrm{z}} ; \tau_{\mathrm{xz}}=\frac{\widetilde{\mathrm{E}}}{2(1+v)} \gamma_{\mathrm{xz}} ; \tau_{\mathrm{yz}}=\frac{\widetilde{\mathrm{E}}}{2(1+v)} \gamma_{\mathrm{yz}} .
\end{gathered}
$$

where $\mu$ is the Poisson's ratio of the shell material, which is assumed to be constant; $\widetilde{\mathrm{E}}_{\mathrm{K}}$ is operator elastic modulus of a conical shell and an edge

$$
\widetilde{E}_{k}[f(t)]=E_{0 \kappa}\left[f(t)-\int_{0}^{t} R_{E \kappa}(t-\tau) f(\tau) d \tau\right]
$$

here $E_{0 \kappa}$ is instantaneous Young's modulus of elasticity $(\kappa=1,2,3 \ldots \mathrm{L}) ; \kappa=1$ is instantaneous modulus of elasticity of the shell, $\mathrm{\kappa}=2,3 \ldots \mathrm{L}$ is instantaneous elastic modulus of the ribs, $f(t)$ is continuous function; $R_{\mathrm{EK}}(t-\tau)$ is the core of relaxation.

The physical relations, taking into account the creep of the material based on the linear theory of heredity, take the form [11

$$
\begin{gathered}
\sigma_{\mathrm{x}}=\frac{\mathrm{E}_{0}}{1-v^{2}}\left[\varepsilon_{\mathrm{x}}^{\mathrm{z}}+v_{1} \varepsilon_{\mathrm{y}}^{\mathrm{z}}-\int_{0}^{\mathrm{t}}\left(\varepsilon_{\mathrm{x}}^{\mathrm{z}}+v_{1} \varepsilon_{\mathrm{y}}^{\mathrm{z}}\right) \mathrm{R}_{\mathrm{E} 1}(\mathrm{t}-\tau) \mathrm{d} \tau\right] \\
\sigma_{\mathrm{y}}=\frac{\mathrm{E}_{0}}{1-v^{2}}\left[\varepsilon_{\mathrm{y}}^{\mathrm{z}}+v_{1} \varepsilon_{\mathrm{x}}^{\mathrm{z}}-\int_{0}^{\mathrm{t}}\left(\varepsilon_{\mathrm{y}}^{\mathrm{z}}+v_{1} \varepsilon_{\mathrm{x}}^{\mathrm{z}}\right) \mathrm{R}_{\mathrm{E} 1}(\mathrm{t}-\tau) \mathrm{d} \tau\right] ; \\
\tau_{\mathrm{xy}}=\frac{\mathrm{E}_{0}}{2(1+v)}\left[\gamma_{\mathrm{xy}}^{\mathrm{z}}-\int_{0}^{\mathrm{t}} \gamma_{\mathrm{xy}}^{\mathrm{z}} \mathrm{R}_{\mathrm{E} 2}(\mathrm{t}-\tau) \mathrm{d} \tau\right] \\
\tau_{\mathrm{zx}}=\frac{\mathrm{E}_{0}}{2(1+v)}\left[\gamma_{\mathrm{zx}}^{\mathrm{z}}-\int_{0}^{\mathrm{t}} \gamma_{\mathrm{zx}}^{\mathrm{z}} \mathrm{R}_{\mathrm{E} 2}(\mathrm{t}-\tau) \mathrm{d} \tau\right] \\
\tau_{\mathrm{yz}}=\frac{\mathrm{E}_{0}}{2(1+v)}\left[\gamma_{\mathrm{yz}}^{\mathrm{z}}-\int_{0}^{\mathrm{t}} \gamma_{\mathrm{yz}}^{\mathrm{z}} \mathrm{R}_{\mathrm{E} 2}(\mathrm{t}-\tau) \mathrm{d} \tau\right] .
\end{gathered}
$$

Here $R_{E 1}(t-\tau), R_{E 2}(t-\tau)$ are relaxation cores. The influence of edge stiffness is taken into account using the Dirac impulse function. The location and height of the edges is set by the function

$$
\mathrm{H}(\mathrm{x}, \mathrm{y})=\sum_{\mathrm{j}=1}^{\mathrm{m}} \mathrm{h}^{\mathrm{j}} \bar{\delta}\left(\mathrm{x}-\mathrm{x}_{\mathrm{j}}\right)+\sum_{\mathrm{i}=1}^{\mathrm{n}} \mathrm{h}^{\mathrm{i}} \bar{\delta}\left(\mathrm{y}-\mathrm{y}_{\mathrm{i}}\right)-\sum_{\mathrm{i}=1}^{\mathrm{n}} \quad \sum_{\mathrm{j}=1}^{\mathrm{m}} \mathrm{h}^{\mathrm{i} j} \bar{\delta}\left(\mathrm{x}-\mathrm{x}_{\mathrm{j}}\right) \bar{\delta}\left(\mathrm{y}-\mathrm{y}_{\mathrm{i}}\right)
$$

Integrating voltages (4) by $Z$ within the range from $-\frac{\mathrm{h}}{2}$ to $\frac{\mathrm{h}}{2}+\mathrm{H}$, we obtain the forces, moments, and transverse forces reduced to the median surface of the shell for the unit length of the median surface

$$
\begin{gathered}
\mathrm{N}_{\mathrm{x}}=\widetilde{\mathrm{G}}_{1}\left[(\mathrm{~h}+\overline{\mathrm{F}}) \cdot \varepsilon_{1}+\overline{\mathrm{S}} \psi_{1}\right] ; \mathrm{N}_{\mathrm{y}}=\widetilde{\mathrm{G}}_{2}\left[(\mathrm{~h}+\overline{\mathrm{F}}) \cdot \varepsilon_{2}+\overline{\mathrm{S}} \psi_{2}\right] ; \\
\mathrm{N}_{\mathrm{xy}}=\widetilde{\mathrm{G}}_{12}\left[(\mathrm{~h}+\overline{\mathrm{F}}) \gamma_{\mathrm{xy}}+\overline{\mathrm{S}} \psi_{12}\right] ; \quad \mathrm{M}_{\mathrm{x}}=\widetilde{\mathrm{G}}_{1}\left[\overline{\mathrm{S}} \varepsilon_{1}+\left(\frac{\mathrm{h}^{3}}{12}+\overline{\mathrm{J}}\right) \psi_{1}\right] ; \\
\mathrm{M}_{\mathrm{y}}=\widetilde{\mathrm{G}}_{2}\left[\overline{\mathrm{S}} \varepsilon_{2}+\left(\frac{\mathrm{h}^{3}}{12}+\overline{\mathrm{J}}\right) \cdot \Psi_{2}\right], \mathrm{M}_{\mathrm{xy}}=\widetilde{\mathrm{G}}_{12}\left[\overline{\mathrm{S}} \gamma_{\mathrm{xy}}+\left(\frac{\mathrm{h}^{3}}{12}+\overline{\mathrm{J}}\right) \Psi_{12}\right] \\
\mathrm{Q}_{\mathrm{x}}=\mathrm{k} \widetilde{\mathrm{G}}_{13}(\mathrm{~h}+\overline{\mathrm{F}}) \cdot\left(\psi_{\mathrm{x}}+\frac{\partial \mathrm{W}}{\partial \mathrm{x}}\right), \mathrm{Q}_{\mathrm{y}}=\mathrm{k} \widetilde{\mathrm{G}}_{23}(\mathrm{~h}+\overline{\mathrm{F}})\left(\psi_{\mathrm{y}}+\frac{1}{\mathrm{x} \sin \theta} \frac{\partial \mathrm{W}}{\partial \mathrm{y}}+\frac{\operatorname{ctg} \theta}{\mathrm{x}} \mathrm{V}\right)
\end{gathered}
$$

Where

$$
\begin{gathered}
\varepsilon_{1}=\varepsilon_{\mathrm{xx}}+v \varepsilon_{\mathrm{yy}}, \quad \varepsilon_{2}=\varepsilon_{\mathrm{yy}}+v \varepsilon_{\mathrm{xx}}, \Psi_{1}=\chi_{1}+v \chi_{2}, \Psi_{2}=\chi_{2}+v \chi_{1}, \Psi_{12}=2 \chi_{12}, \\
\widetilde{\mathrm{G}}_{1}[\mathrm{f}(\mathrm{t})]=\widetilde{\mathrm{G}}_{2}[\mathrm{f}(\mathrm{t})]=\frac{\widetilde{\mathrm{E}}}{1-v^{2}}[\mathrm{f}(\mathrm{t})]=\frac{\mathrm{E}_{0}}{1-v^{2}}\left(\mathrm{f}(\mathrm{t})-\int_{0}^{\mathrm{t}} \mathrm{R}_{\mathrm{E}}(\mathrm{t}-\tau) \mathrm{f}(\tau) \mathrm{d} \tau\right),
\end{gathered}
$$




$$
\widetilde{G}_{12}[f(t)]=\widetilde{G}_{13}[f(t)]=\widetilde{G}_{23}[f(t)]=\frac{\widetilde{E}[f(t)]}{2(1+v)}=\frac{E_{0}}{2(1+v)}\left(f(t)-\int_{0}^{t} R_{E}(t-\tau) f(\tau) d \tau\right)
$$

$\bar{F}, \bar{S}, \bar{J}$ are the areas (transverse or longitudinal) of the cross-section of the edges per unit length of the median surface. form

The static moment and the moment of inertia of the median surface of the shell have the

$$
\bar{F}=\int_{h / 2}^{h / 2+H} d z \quad ; \quad \bar{S}=\int_{h / 2}^{h / 2+H} z d z ; \quad \bar{J}=\int_{h / 2}^{h / 2+H} z^{2} d z .
$$

Let a transverse dynamic load act on the shell $q(x, y, t)$. Then the unknown desired offset functions are $U, V, W$ and the angles of rotation of the normal $\psi_{x}, \psi_{y}$ are functions of variables $x, y$, and $t$. The functional of the total strain energy of the viscoelastic shell has the form

$$
J=\int_{t_{0}}^{t_{1}}(\mathrm{~K}-\Pi+A) d t
$$

Here $K$ is kinetic energy of the system, $\Pi$ is potential energy of the system, $A$ is the work of external forces, where

$$
\begin{gathered}
\mathrm{K}=\frac{\rho}{2} \int_{a_{1}}^{a} \int_{0}^{b} \int_{-\frac{h}{2}}^{\frac{h}{2}+H}\left[\left(\dot{u}^{z}\right)^{2}+\left(\dot{v}^{z}\right)^{2}+\left(\dot{w}^{z}\right)^{2}\right] x \sin \theta d x d y d z= \\
=\frac{\rho}{2} \int_{a_{1}}^{a} \int_{0}^{b}\left[(h+\bar{F})\left(\dot{u}^{2}+\dot{v}^{2}+\dot{w}^{2}\right)+2 \bar{S}\left(\dot{u} \dot{\psi}_{x}+\dot{v} \dot{\psi}_{y}\right)+\right. \\
\left.\quad+\left(\frac{h^{3}}{12}+\bar{J}\right)\left(\dot{\psi}_{x}^{2}+\dot{\psi}_{y}^{2}\right)\right] x \sin \theta d x d y \\
\exists=\Pi-A=\frac{1}{2} \int_{a_{1}}^{a} \int_{o}^{b}\left[N_{x} \varepsilon_{x x}+N_{y} \varepsilon_{y y}+N_{x y} \gamma_{x y}+M_{x} \chi_{1}+M_{y} \chi_{2}+2 M_{x y} \chi_{12}+\right. \\
\left.+Q_{x}\left(\psi_{x}+\frac{\partial w}{\partial x}\right)+Q_{y}\left(\psi_{y}+\frac{1}{x \sin \theta} \cdot \frac{\partial w}{\partial y}+\frac{\operatorname{ctg} \theta}{x} v\right)-2 q w\right] x \sin \theta d x d y
\end{gathered}
$$

In the above formulas, the density of the shell material. In expressions (9) and (10), the dots denote the derivatives of the variable $t ; b=2 \pi$ is for a conical shell. The energy can be expressed in terms of deformations, then the expression (10) is represented as follows

$$
\begin{gathered}
\exists=\frac{\tilde{E}}{2\left(1-\mu^{2}\right)} \int_{a_{1}}^{a} \int_{0}^{b}\left[( h + \overline { F } ) \left(\varepsilon_{x x}^{2}+\varepsilon_{y y}^{2}+2 \mu \varepsilon_{x x} \varepsilon_{y y}+\mu_{1} \gamma_{x y}^{2}+\frac{5}{6} \mu_{1}\left(\psi_{x}+\frac{\partial w}{\partial x}\right)^{2}+\right.\right. \\
\left.+\frac{5}{6} \mu_{1}\left(\psi_{y}+\frac{1}{x \sin \theta} \frac{\partial w}{\partial y}+\frac{c t g \theta}{x} v\right)^{2}\right)+2 \bar{S}\left(\chi_{1} \varepsilon_{x x}+\chi_{2} \varepsilon_{y y}+\mu \chi_{2} \varepsilon_{x x}+\mu \chi_{1} \varepsilon_{y y}+2 \mu_{1} \gamma_{x y} \chi_{12}\right)+ \\
\left.\quad+\left(\frac{h^{3}}{12}+\bar{J}\right)\left(\chi_{1}^{2}+\chi_{2}^{2}+2 \mu \chi_{1} \chi_{2}+4 \mu_{1} \chi_{12}^{2}\right)-2\left(1-\mu^{2}\right) \frac{q}{E} w\right] x \sin \theta d x d y
\end{gathered}
$$

where $\mu_{1}=\frac{1-v}{2}$ If the conical viscoelastic shell is closed, then $a_{1}=0$.

Consider a reinforced conic shell with narrow edges. The problem under consideration is solved in dimensionless parameters. Then the main relations take the following form

$$
\begin{gathered}
\xi=\frac{x}{a}, \quad \eta=\frac{y}{b}, \quad \lambda=\frac{a}{b x \sin \theta}, \quad \lambda=\frac{\lambda_{1}}{\xi}, \quad \bar{U}=\frac{a u}{h^{2}}, \\
\bar{V}=\frac{b x \sin \theta v}{h^{2}}, \quad \bar{W}=\frac{w}{h}, \quad \bar{\psi}_{x}=\frac{a \psi_{x}}{h}, \quad \bar{\psi}_{y}=\frac{b x \sin \theta \psi_{y}}{h},
\end{gathered}
$$




$$
\bar{a}=\frac{a}{h}, \quad \bar{P}=\frac{a^{4} P}{E h^{4}}, \quad \bar{t}=\frac{h}{a^{2}} \sqrt{\frac{E_{0}}{\left(1-v^{2}\right) \rho}} t, \quad \bar{F}=\frac{\bar{F}}{h}, \bar{J}=\frac{\bar{s}}{h^{2}}, \quad \bar{J}=\frac{\bar{J}}{h^{3}},
$$

and from here, we get the following expressions for the kinetic and potential energies

$$
\begin{gathered}
\overline{\mathrm{K}}=\frac{1}{\bar{a}^{2}} \int_{a_{1}}^{1} \int_{0}^{1}\left[(1+\overline{\bar{F}})\left(\dot{\bar{U}}^{2}+\lambda^{2} \dot{\bar{V}}^{2}+\bar{a}^{2} \dot{\bar{W}}^{2}\right)+2 \overline{\bar{S}}\left(\dot{\bar{U}} \dot{\bar{\psi}}_{x}+\lambda^{2} \dot{\bar{V}}_{\overline{\bar{\psi}}}\right)+\right. \\
\left.+\left(\frac{1}{12}+\overline{\bar{J}}\right)\left(\dot{\bar{\psi}}_{x}^{2}+\lambda^{2} \dot{\bar{\psi}}_{y}^{2}\right)\right] \xi d \xi d \eta \cdot(13) \\
\bar{\jmath}=\int_{\bar{a}_{1}}^{1} \int_{0}^{1}\left[( 1 + \overline { \overline { F } } ) \left(\bar{\varepsilon}^{2}+\lambda^{4} \bar{\varepsilon}_{y y}^{2}+2 v \lambda^{2} \bar{\varepsilon}_{x x} \bar{\varepsilon}_{y y}+\mu_{1} \lambda^{2} \bar{\gamma}_{x y}^{2}+\frac{5}{6} \mu_{1} \bar{a}^{2} \cdot\left(\bar{\psi}_{x}+\frac{\partial \bar{W}}{\partial \xi}\right)^{2}+\right.\right. \\
\left.+\frac{5}{6} \mu_{1} \lambda^{2} \bar{a}^{2} \cdot\left(\bar{\psi}_{y}+\frac{\partial \bar{W}}{\partial \eta}+\frac{c_{3}}{\xi} \bar{V}\right)^{2}\right)+2 \overline{\bar{S}}\left(\bar{\chi}_{1} \bar{\varepsilon}_{x x}+\lambda^{4} \bar{\chi}_{2} \bar{\varepsilon}_{y y}+v \lambda^{2} \bar{\chi}_{2} \bar{\varepsilon}_{x x}+v \lambda^{2} \bar{\chi}_{1} \bar{\varepsilon}_{y y}+\right. \\
\left.2 \mu_{1} \lambda^{2} \bar{\gamma}_{x y} \bar{\chi}_{12}\right)+\left(\frac{1}{12}+\bar{J}\right) \cdot\left(\bar{\chi}_{1}^{2}+\lambda^{4} \bar{\chi}_{2}^{2}+2 v \lambda^{2} \bar{\chi}_{1} \bar{\chi}_{2}+4 \mu_{1} \lambda^{2} \bar{\chi}_{12}^{2}\right)- \\
\left.-2\left(1-v^{2}\right) \bar{P} \bar{W}\right] \xi d \xi d \eta .
\end{gathered}
$$

The variational equation for a thin viscoelastic shell supported by 1 -and j-direction edges is obtained as a variation of the sum of the potential and kinetic energies, taking into account the conjugation condition [12]:

$$
\delta K+\sum_{l=1}^{N} \delta K_{l}+\sum_{j=1}^{n} \delta K_{l}+\delta \ni+\sum_{l=1}^{m} \delta \ni_{l}=0, \quad N=n+m,
$$

where $\ni_{l}=\Pi_{l}-A_{l}$ - the potential difference of external forces and work applied to the edges. This sum contains as many terms of equations (15) as there are edges in the corresponding directions. Thus, about the considered ribbed shell, the Lagrange principle can be formulated as follows [13-15]:

-actual displacements of the median surface of the shell $u, v, w$ and edges $\mathrm{u}_{m}, v_{m}, w_{m}(m=k, j)$, corresponding to these boundary conditions and the load, and transferring the shell from its natural position to the state of elastic equilibrium, differ from all possible displacements in that they give the system under consideration the minimum amount of potential energy.

The natural oscillations of a conical shell with a freely supported end face are considered. Thus the mathematical formulation of the problem is formulated, and the main relations of viscoelastic conical shells under natural vibrations are given, which take into account the geometric nonlinearity, the discrete introduction of viscoelastic edges, their shear, and torsional stiffness, transverse shifts, and inertia of rotation.

2.2. Methods of solution

It is assumed that the integral terms in (4) -(6) are small. Then for the function $f(t)$ there is a function $f(t)=\phi(t) e^{-i \omega_{R} t}$ and the integral terms are replaced by the following expressions [16]

$$
\bar{E}_{\mathrm{K}} \phi=E_{0 \kappa}\left[1-\Gamma_{\mathrm{K}}^{\mathrm{C}}\left(\omega_{R}\right)-i \Gamma_{\mathrm{K}}^{S}\left(\omega_{R}\right)\right] \phi
$$

where

$$
\Gamma^{C}\left(\omega_{R}\right)=\int_{0}^{\infty} R_{\mathrm{E}}(\tau) \cos \omega_{R} \tau d \tau, \Gamma^{s}\left(\omega_{R}\right)=\int_{0}^{\infty} R_{\mathrm{E}}(\tau) \sin \omega_{R} \tau d \tau-
$$

responsible for the cosine and sine of the Fourier transform, $\omega_{R}$ is the actual value. The Koltunov - Rzhanitsyn three-parameter kernel is used in the calculations: 


$$
R_{k}(t)=A_{k} e^{-\beta_{k} t} / t^{1-\alpha_{k}}
$$

To calculate the dynamic characteristics of truncated conic shells, in (1)-(4), we neglect the nonlinear terms that take into account geometric nonlinearities and use the finite element method (FEM) in displacements. Let us consider 8-node isometrically curved finite elements (FE), the so-called "degenerate" element of the shell [16, 17]. The element is designed for calculating shells of medium and small thickness with edges. The GE geometry represents a curved parallelepiped in three-dimensional space with a linear surface in thickness. Used local $\xi, \eta, \zeta$, and the global Cartesian $\mathrm{x}, \mathrm{y}, \mathrm{z}$ coordinate systems. The coordinates of an arbitrary CE point are expressed in terms of the coordinates of the node points $\bar{r}_{i}$ and components of the unit normal vector $\bar{n}_{i}$. Finite element representation of the equilibrium equations of a finite element system in a state of motion, taking into account (5), (6), (9), and (15) has the form [18-15].

$$
[M]\{\ddot{q}\}+\left[\bar{K}\left(\omega_{R}\right)\right]\{q\}+\{R\}=0
$$

where $[M]=\sum_{i, j=1}^{n}\left[m_{i j}\right]$ is mass matrix of the system $\left(\left[m_{i j}\right]=\alpha_{1}\left[m_{i j}\right]_{a}+\beta_{1}\left[m_{i j}\right]_{p}\right.$, $\left[m_{i j}\right]_{a}$ are elements of the mass matrix of the truncated conic shell, $\left[m_{i j}\right]_{p}$ is elements matrix of the mass of the reinforcing rod, $\left(\alpha_{1}, \beta_{1}\right.$ are dimensionless coefficients), $\{R\}$ is the vector of external loads, $\left[\bar{K}\left(\omega_{R}\right)\right]$ is the stiffness matrix of the system, $\left([K]=\sum_{i, j=1}^{n}\left[k_{i j}\right]\right.$ is matrix of rigidity of the panel of the conical shell and the reinforcing rod), $\{q\}$ is unknown nodal movements, $\{R\}$ is vector of external loads. The mass matrix (16) is consistent: the structure of the mass matrix $[M]=\sum_{i, j=1}^{n}\left[m_{i j}\right]$ completely coincides with the structure of the stiffness matrix. Both matrices $\left([M],\left[\bar{K}\left(q^{t}, \omega_{R}\right)\right]\right)$ have the size $(\mathrm{NxN})$, which corresponds to the number of degrees of freedom of the CE.

It is assumed that $\{R\}=0$, then the eigenvalues of the truncated conic shell are considered. The solution of equation (16) is sought in the form

$$
\{q\}=\left\{Q_{A}\right\} e^{-i \omega t}
$$

where $\left\{Q_{A}\right\}$ is the amplitude of unknown nodal displacements, a complex value; $\omega=\omega_{R}+i \omega_{I}$ is the complex frequency to be determined.

Substituting (17) into (16), we obtain the following homogeneous algebraic equation

$$
\left(-\omega^{2}[M]+\left[K\left(\omega_{R}, q^{\circ}\right)\right]\right)\left\{Q_{A}\right\}=0 .
$$

The complex roots of the frequency equation (18) are determined by the Muller method; at each iteration of the Muller method, the Gauss method is used with the main element selection $[21,22]$. 


\section{Results and Discussion}

As input, you should set a radius (large and small), the height and thickness of the shell, the angle of the floor of the solution of a truncated cone, elastic modulus, Poisson's ratio, the kernel parameters of the relaxation of the material and the geometric and mechanical parameters ribs $\left(R_{E p}(t)=0\right)$. As the relaxation core of the viscoelastic shell material, we take a three-parameter core $R_{E 0}(t)=\frac{A e^{-\beta t}}{t^{1-\alpha}}$ Rzhanitsyna - Koltunova [23,24,25], having a weak singularity. Here $A, \alpha, \beta$-the materials parameters. The natural oscillations of the pinched truncated conical shell from the stringers (ribs) were experimentally studied in the work of Watkins and Clery in 1964. The results of the comparison are shown in Figure 2. Figure 2 shows: 1, 2-the theoretical results obtained by us at $1 . \theta=75^{\circ} ; 2 . \theta=60^{\circ}$, $R_{1} / R_{2}=0.8 ; \varepsilon=1.3 \cdot 10^{-5}, \circ, *$ - the results of the experiment.

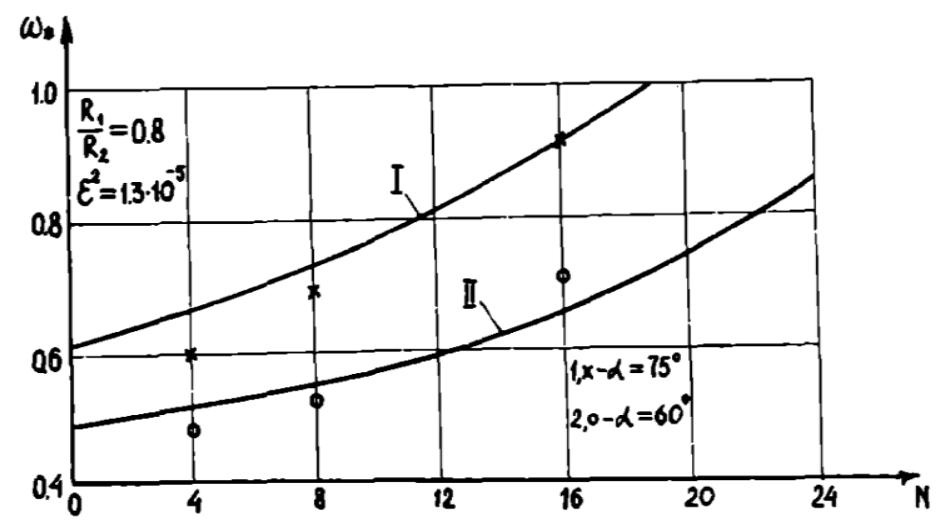

Fig.2. Changing the frequency parameter $\left(R_{E 0}=0\right)$ the truncated conic shell of rotation as a function of $\mathrm{N}$ under different conditions $\gamma: 1 . \theta=75^{\circ} ; 2 . \theta=60^{\circ}$.

Viscoelastic truncated conical shells are considered, in which the large bases are pivotally supported, and the second bases are freely supported on the conical shell. The conical shell is supported by 4 ribs, $4 \mathrm{~h}$ high and $2 \mathrm{~h}$ wide (the lengths of the arcs between the edges are the same $-s_{i}=\pi a / 2$ ). The parameters of a truncated conical shell made of Plexiglass have the following values: taper angle $\theta=0.20,0.40,0.60,0.80$, radii of the base of the truncated conic shell $-a_{1}=15 \mathrm{M}, \quad \mathrm{a}=18 \mathrm{M}$ (the length of the shell is 10 meters). The physical and mechanical characteristics of the rib and shell, respectively, take the following values: $\rho_{\mathrm{c}}=7,8 \cdot 10^{3} \frac{\mathrm{K} \Gamma}{\mathrm{M}^{3}}, \rho_{0}=3 * 10^{3} \frac{\mathrm{K} \Gamma}{\mathrm{M}^{3}}, v_{\mathrm{c}}=0.25, v_{0}=0.35, \mathrm{E}_{\mathrm{c}}=2 \times 10^{12}$ $\mathrm{hPa}, \mathrm{E}_{0}=20 \times 10^{12} \mathrm{~Pa}$. The values of the rheological parameters of the shell are taken as $A=0,048 ; \quad \beta=0,05 ; \quad \alpha=0,1$. Table 1 shows the complex values of the low frequencies of a reinforced (with four rods) truncated conical shell at different shell thicknesses in the limit of the Kirchhoff - Love hypothesis [26-28]. 
Table 1. Frequency change depending on the thickness of the shell

\begin{tabular}{|c|c|c|c|}
\hline \multirow[t]{2}{*}{$\mathrm{n}$} & \multirow[t]{2}{*}{$\mathrm{h}$} & \multicolumn{2}{|c|}{$\omega=\omega_{R}+i \omega_{I}$} \\
\hline & & $\omega_{R}$ & $-\omega_{I}, 10^{-2}$ \\
\hline 1 & \multirow{4}{*}{0.05} & 1.94894 & 1.77381 \\
\hline 2 & & 2.18942 & 2.57321 \\
\hline 3 & & 3.44160 & 2.90566 \\
\hline 4 & & 3.92574 & 3.04574 \\
\hline 1 & \multirow{4}{*}{0.02} & 1.31147 & 1.42621 \\
\hline 2 & & 1.53439 & 2.49433 \\
\hline 3 & & 3.54578 & 2.98778 \\
\hline 4 & & 4.12429 & 3.21429 \\
\hline 1 & \multirow{4}{*}{0.01} & 1.05218 & 1.22718 \\
\hline 2 & & 1.26867 & 1.36860 \\
\hline 3 & & 3.55691 & 3.45697 \\
\hline 4 & & 4.53974 & 3.65924 \\
\hline
\end{tabular}

It is necessary to determine the values of the complex natural frequency and the corresponding oscillation forms when both ends of the shell contour are pivotally supported $\left(N_{1}=M_{1}=U=V=W=0\right)$. The axisymmetric oscillations correspond to the minimum eigenfrequencies (the real parts of the complex frequencies). Analysis of the calculation results allows us to conclude that with a decrease in the thickness of the viscoelastic conical shell, its real and imaginary parts of the first and second oscillation frequencies monotonically decrease. The change in the imaginary part of the frequencies from the number of edges is shown in Figure 3. As can be seen from figure 3, the reinforcement of the shell with four longitudinal edges allows you to increase the imaginary parts of the natural frequency of the truncated conical shell. Parameter $\gamma=0$ - corresponds to a reinforced cylindrical shell, $\theta=0.20,0.40,0.60,0.80$ - correspond to the angle of taper of the reinforced truncated conical shell.

Considering the rheological properties of the material allows you to increase (or decrease) the frequency values of the shell up to $10 \%$. For bending vibrations, a significant decrease in the local maxima of normal displacements is observed with an increase in the area of the annular edges of the shell $[29,30]$.

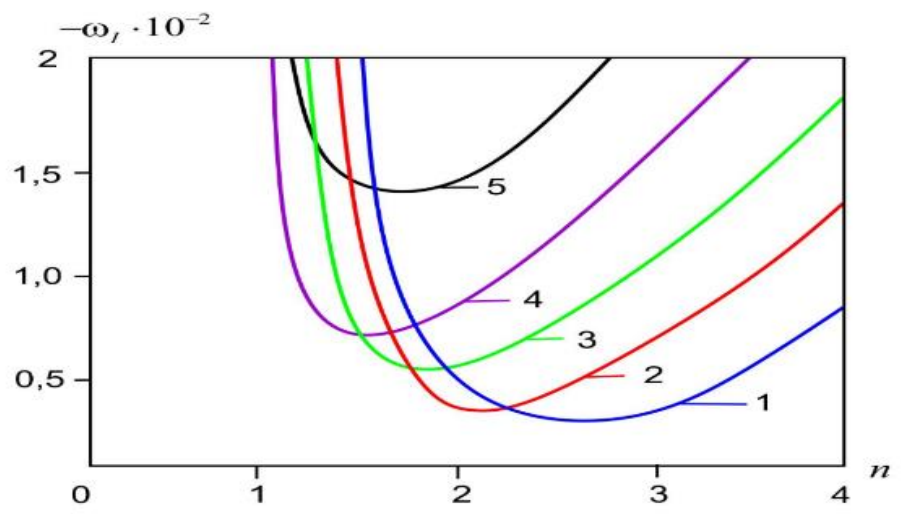

Fig.3. Change of the parameter (imaginary part) of the complex frequency of the truncated conic shell as a function of the number of edges $\mathrm{n}$ for different $\gamma: 1 . \theta=0 ; 2$. $\theta=0.20 ; 3 . \theta=0.40 ; 4 . \theta=0.60 ; 5$. $\theta=0.80$.

This effect becomes more noticeable with an increase in the frequency number $\mathrm{n}[31,32]$. 


\section{Conclusions}

1. An algorithm for solving the problem of natural vibrations of ribbed viscoelastic conical shells is developed. The finite element method, the freezing method, and the Muller and Gauss methods are used to solve dynamic problems.

2. Analysis of the calculation results shows that with a decrease in the thickness of the viscoelastic conical shell, the real and imaginary parts of the first and second oscillation frequencies monotonically decrease. The real parts of the third and fourth frequencies decrease moderately, and the corresponding imaginary parts gradually increase. The minimum value of the attenuation decrement as a function of the number of edges (n) of the reinforced shell is found. Considering the rheological properties of the material allows you to increase the frequency values of the shell to $10 \%$.

\section{Reference}

1. Novojilov V. V, Tonkix obolochek doctrina. (1, Sudpromizdat) p 431. (1962).

2. Volmir A S, Nelineynaya viribus propriis laminas bellicorum bombos imitantur (M. Nauka) p CDXXXII. (1972).

3. Rjanitsyn R.A, Stroitelnaya mechanicis tractatur (M: Vysshaya shkola) CD. (1982).

4. Sheremeteva A K and Chexonin K A. Deformationis polymeric analysis de compositum de proprietatibus et conditionibus tempus fluminum liberam et animi remis Acta Congressus Internationalis materiāli XXI Latini Opera Application Systems (VMSPPS'2019), 24-31 Maii MMXIX, Alushta M Vo-Izd MAI pp 365-367, (2019).

5. Mirsaidov M M, Safarov I I and Teshaev M Kh, Dynamics Structurally inhomogeneous lamellae intertexta, et ex Mechanica Crusta Systems Aequationes Mathematicae parte 1.Journal et I. 7 (10), October XIV, MMXIX.

6. Teshaev M.K, Safarov I.I, Kuldashov N.U, Ishmamatov M.R and Ruziev T.R, On the Distribution of Free Waves on the Surface of a Viscoelastic Cylindrical, Cavity Journal of Vibrational Engineering and Technologies 8 (4) pp 579-585.

7. Makowski J, Pietraszkiewicz W and Stumpf H, On the general form of jump conditions for thin irregular shells Arch. Mech. 50, (3). pp 483-495 (1998).

8. Mirsaidov M M, Safarov I I and Teshaev M Kh, Dynamics of StructuralInhomogeneous Laminate and Shell Mechanical Systems with Point Constraints and Focused Masses Part 2 Statement of the Problem of Forced Oscillations, Methods of Solution, Computational Algorithm and Numerical Results Journal of Applied Mathematics and Physics. 7 (11). November 5. (2019).

9. Safarov I I, Teshaev M Kh and Boltaev Z I, Own Vibrations of Bodies Interacting with Unlimited Deformable Environment, Open Access Library Journal 5, pp 1-22 (2018).

10. Safarov I I, Teshaev M Kh and Akhmedov M S, Free Oscillations of a Toroidal Viscoelastic Shell with a Flowing Liquid, American Journal of Mechanics and Applications 6 (2) pp 37-49 (2018).

11. Abovskiy N P, Rebristye obolochki (Krasnoyarsk) p 61. (1967),

12. Ershov C.P, Sostoyanie ego perspektivy razvitiya raschetno-eksperimentalnyx rabot in area proektirovaniya tonkostennyx ix konstruktsiy kompozitsionnyx materialov Mexanika kompozitnyx materialov 1. pp 86-92. (1988). 
13. Efimov.A.B, Aksenenko V.S and Tsvelix V.A, Reshenie osesimmetrichnoy zadachi teorii uprugosti dlya nesjimaemyx materialov s pomoshchyu hybridnogo metoda konechnyx elementov, Matematicheskoe modelirovanie sistem protsessov. I (I) pp 67-81. (1992)

14. Laura P A and Gutierrez R H Vibration analysis on a rectangular plate subjected to a thermal gradient Journalof Sound and Vibration 72 pp 263-264 (1980)

15. Gupta A K. and Khanna A, Vibration of clamped viscoelastic rectangular plate with parabolic thickness variations Shock and Vibration 15 (6) pp 713-723 (2008)

16. Maiboroda V P, Troyanovskii I E, Safarov I I, Vazagashvili M G and Katalymova I $\mathrm{V}$, Wave attenuation in an elastic medium Journal of Soviet Mathematics 60 (2). pp 1379-1382. (1992)

17. Lal R, Transverse vibrations of orthotropic non-uniform rectangular plate with continuously varying density Indian, Journal of Pure and Applied Mathematics 34 pp 587-6062003

18. Sobotka Z, Free vibration of visco-elastic orthotropic rectangular plates Acta Technica CSAV. 6 pp 678-705, (1978)

19. Singh B and Saxena V, Transverse vibration of rectangular plate with bi-directional thickness variation Journal of Sound and Vibration 198 pp 51-65. (1996).

20. Safarov I I, Homidov F F, Rakhmonov B S and Almuratov S N, Seismic vibrations of complex relief of the surface of the naryn canyon (on the Norin river in Kyrgyzstan) during large-scale underground explosions, Journal of Physics Conference Series 1706 (1) 012125 (2020)

21. Kaplunov J D and Wilde M V, Edge and interfacial vibrations in elastic shells of revolution, J. Appl. Math. Phys. (ZAMP) 51 pp 29-48 (2000)

22. Lekomtsev S.V, Konechno-elementnye algoritmy rascheta sobstvennykh kolebanii trekhmernykh obolochek Vychisl. mekh. splosh. Sred 5 (2) pp 233-243 (2012).

23. Safarov I I, Teshaev M Kh and Boltayev Z I. Propagation of linear waves in multilayered structural - Inhomogeneous cylindrical shells Journal of Critical Reviews 7 (12) pp 893-904 (2020).

24. Bochkarev S A and Matveenko V.P, Ustoichivost' koaksial'nykh tsilindricheskikh obolochek, soderzhashchikh vrashchayushchiisya potok zhidkosti Vychisl. mekh. splosh. sred 6 (1) pp 94-102 (2013).

25. Maiboroda V P, Safarov I I and Troyanovskii I E, Free and forced oscillations of a system of rigid bodies on inhomogeneous viscoelastic snubbers, Soviet Machine Science (English Translation of Mashinovedenie) (3) pp 25-31 (1983)

26. Mirsaidov M, Safarov I, Boltayev Z and Teshaev M, Spread waves in a viscoelastic cylindrical body of a sector cross section with cutouts IOP Conference Series: Materials Science and Engineering 869 (4) 042011 (2020).

27. Prisekin L V and Rastorguev I G. Nova elementa modum finalis enim in mechanicis deformiruemyx Tel Novosibirsk NGTU CCXXXVIII p. (2010).

28. Safarov I I, Teshaev M, Toshmatov E, Boltaev Z and Homidov.F F, Torsional vibrations of a cylindrical shell in a linear viscoelastic medium IOP Conference Series: Materials Science and Engineering 883 (1) 012190 (2020).

29. Mirsaidov MM, Safarov I.I, Teshaev M.K and Boltayev Z I, Dynamics of structural Inhomogeneous coaxial-multi-layered systems cylinder-shells Journal of Physics: Conference Series 1706 (1) 012033 (2020)

30. Teshaev M Kh, Safarov I I and Mirsaidov M M, Oscillations of multilayer viscoelastic composite toroidal pipes Journal of the Serbian Society for Computational Mechanics 13 (2) pp 104-115 (DOI: 10.24874 / jsscm.2019.13.02.02.08) (2019) 
31. Mirsaidov M, Safarov I I and Teshaev M Kh, Dynamic instability of vibrations of thin-wall composite curvorine viscoelastic tubes under the influence of pulse pressure E3S Web of Conferences 16414013 (2020).

32. Boltaev Z I, Safarov I I and Razokov T, Natural vibrations of spherical inhomogeneity in a viscoelastic medium International Journal of Scientific and Technology Research. 9 (1). pp 3674-3680 (2020), 